TAIWANESE JOURNAL OF MATHEMATICS

Vol. 13, No. 2A, pp. 493-500, April 2009

This paper is available online at http://www.tjm.nsysu.edu.tw/

\title{
ALMOST SOLUTIONS OF EQUATIONS IN PERMUTATIONS
}

\author{
Lev Glebsky and Luis Manuel Rivera
}

\begin{abstract}
We will say that the permutations $f_{1}, \ldots, f_{n}$ are an $\epsilon$-solution of an equation if the normalized Hamming distance between its 1.h.p. and r.h.p. is $\leq \epsilon$. We give a sufficient conditions when near to an $\epsilon$-solution exists an exact solution and some examples when there does not exist such a solution.
\end{abstract}

\section{Introduction and Formulation of Main Results}

Let $S_{n}$ denote the group of all permutations of the finite set $\{n\}=\{1, \ldots, n\}$. For $f, g \in S_{n}$ let $h(f, g)=\frac{|\{a:(a) f \neq(a) g\}|}{n}$. It is easy to check that $h(\cdot, \cdot)$ is a biinvariant metric on $S_{n}$ [2]. In the article we are going to study almost solutions of equations in $S_{n}$. For example, fix $p \in \mathbf{N}$ and for $f \in S_{n}$ consider equation

$$
x^{p}=f .
$$

Such an $x$, if exists, is called to be $p$-root of $f$. Not every $f \in S_{n}$ has a $p$-root, but any $f \in S_{n}$ has an almost $p$-root for sufficiently large $n$. Precisely, the following theorem is true

Theorem 1. For any $p \in \mathbf{N}$ there exists a sequence $\delta_{n}>0, \lim _{n \rightarrow \infty} \delta_{n}=0$ such that for any $f \in S_{n}$ there exists a permutation $g \in S_{n}$ with $h\left(g^{p}, f\right) \leq \delta_{n}$.

One of the motivations to consider such a question is studying sofic groups, a class of groups that was introduced by Gromov and Weiss [6, 12]. Later G. Elek and E. Szabó [3] define a family of sofic groups that they called universal sofic groups. Every sofic group is isomorphic to a subgroup of an universal sofic group.

Theorem 1 imply the following

Received September 14, 2007, accepted September 15, 2007.

Communicated by Valentin Afraimovich.

2000 Mathematics Subject Classification: 20B30, 20B99.

Key words and phrases: Permutations, Equations, Sofic groups. 
Corollary 1. An universal sofic group $\mathcal{U}$ is an $\mathbf{N}$-root group. In other words: any $g \in \mathcal{U}$ has a $p$-root for any $p \in \mathbf{N}$.

The other set of questions is about stability of a system of equations in permutations. In order to give the precise formulation of the problem we present the following definitions. Let $w\left(x_{1}, \ldots, x_{k}\right), u\left(x_{1}, \ldots, x_{k}\right)$, be expressions using $x_{j}$, $x_{j}^{-1}$ and multiplications (due to associativity we may think that $w, u$ are words in $\left.\left\{x_{1}, x_{1}^{-1}, \ldots, x_{k}, x_{k}^{-1}\right\}\right)$.

\section{Definition 1.}

1. We say that permutations $f_{1}, . ., f_{k}$ are an $\epsilon$-solution of an equation ( $\epsilon$-satisfy an equation) $w\left(x_{1}, \ldots, x_{k}\right)=u\left(x_{1}, \ldots, x_{k}\right)$, iff $h\left(w\left(f_{1}, \ldots, f_{k}\right), u\left(f_{1}, \ldots, f_{k}\right)\right) \leq \epsilon$

2. We say that permutations $f_{1}, . ., f_{k}$ are an $\epsilon$-solution of a system ( $\epsilon$-satisfy a system) of equations

$$
w_{i}\left(x_{1}, \ldots, x_{k}\right)=u_{i}\left(x_{1}, \ldots, x_{k}\right), \quad i=1, . ., r
$$

iff $f_{1}, . ., f_{k} \epsilon$-satisfy every equation of the system.

3. System (2) is called stable (in permutations) iff there exists $\delta_{\epsilon}, \lim _{\epsilon \rightarrow 0} \delta_{\epsilon}=0$ such that for any $\epsilon$-solution $f_{1}, f_{2}, \ldots, f_{k} \in S_{n}$ of the system (2) there exists an exact solution $\tilde{f}_{1}, \tilde{f}_{2}, \ldots, \tilde{f}_{k} \in S_{n}$ of the system (2) such that $d\left(f_{i}, \tilde{f}_{i}\right)<\delta_{\epsilon}$ for $i=1, \ldots, k$. (Note, that $\delta_{\epsilon}$ is independent of $n$.)

There are some relations of stability of the system (2) and the properties of the group $G=\left\langle x_{1}, \ldots, x_{k} \mid w_{i}\left(x_{1}, \ldots, x_{k}\right)=u_{i}\left(x_{1}, \ldots, x_{k}\right), i=1, \ldots, r\right\rangle$.

Theorem 2. Let $G=\left\langle x_{1}, \ldots, x_{k} \mid w_{i}\left(x_{1}, \ldots, x_{k}\right)=u_{i}\left(x_{1}, \ldots, x_{k}\right), i=1, \ldots, r\right\rangle$.

- If the group $G$ is finite then the system (2) is stable in permutations.

- If the group $G$ is sofic but not residually finite, then the system (2) is unstable in permutations.

So, for example the equation $x_{1}^{3}=x_{2}^{-1} x_{1}^{2} x_{2}$ is unstable in permutations because the Baumslag-Soliter group $G=\left\langle x_{1}, x_{2} \mid x_{1}^{3}=x_{2}^{-1} x_{1}^{2} x_{2}\right\rangle$ is sofic but not residually finite $[7,9,10]$. On the other hand the system:

$$
x^{3}=y^{3}=(x y)^{3}=\left(x^{2} y\right)^{3}=i d
$$

is unstable in permutations, because the corresponding group is finite. Of course, in most cases, Theorem 2 says nothing about stability of a system of equations and 
generally the question seems to be very difficult. Particularly, we believe that the commutator relation

$$
x y=y x
$$

is unstable but do not have a proof yet. In [5] the similar but easier question about commutator relation was considered.

The similar questions about matrices was widely studied and solved (at least for commutator relation), see for example $[1,8,11]$. Let us discuss these results in more details. First of all to formulate the problem we can generalize Definition 1 from $S_{n}$ to any family of sets, where metrics and multiplications are defined. Particularly, we may ask $f_{1}, f_{2}, \ldots, f_{k}$ in the definition 1 to be unitary (self-adjoint) matrices, with the metrics $d(A, B)=\|A-B\|,\|X\|=\sup _{\|x\|=1}\|X x\|$. So, we can speak about stability of the system (2) in unitary (self-adjoint) matrices. In this case it is also important that $\delta_{\epsilon}$ is independent of the size of the matrices. The results of $[8,11]$ say that the commutator relation is stable in self-adjoint matrices and unstable in unitary matrices.

Although permutations have natural representations by unitary matrices, instability of the commutator relation for unitary matrices seems to say nothing about stability of the commutator relation in permutations. One of the difficulty here is that the representations of permutations by unitary matrices is not uniformly continuous for the distances defined above. It looks like that the following distances for matrices are more relevant for the study of stability in permutations.

1. $d(A, B)=\|A-B\|_{T}$, where $\|X\|_{T}=\sqrt{\frac{1}{n} \operatorname{trace}\left(X X^{*}\right)}$, or

2. $d(A, B)=\frac{\operatorname{rank}(A-B)}{n}$,

where $n \times n$ is the size of the matrices. We do not know any results about stability of commutator relations in matrices for those distances, but there are some related works around von Neumann algebras, where perturbations by compact operators is considered. (Calkin algebras, essentially normal operators, see [4] and the bibliography in it.)

\section{Proofs of The Theorems}

In this section we present the proofs of theorems 1 and 2 , in order to proof theorem 1 we use two propositions.

Proof of Theorem 1. Some important facts. From the right and left invariance of the metric $d$ its follows that $d\left(x^{n}, y^{n}\right) \leq n d(x, y)$, the proof by induction: $d\left(x^{(n+1)}, y^{(n+1)}\right) \leq d\left(x^{n} x, x^{n} y\right)+d\left(x^{n} y, y^{n} y\right)=d(x, y)+d\left(x^{n}, y^{n}\right)$ 
So, it is enough to prove Theorem 1 for prime $p$. Indeed, if $d\left(f_{1}^{p_{1}}, g\right) \leq \epsilon_{1}$ and $d\left(f_{2}^{p_{2}}, f_{1}\right) \leq \epsilon_{2}$ then $d\left(f_{2}^{p_{1} p_{2}}, g\right) \leq \epsilon_{1}+p_{1} \epsilon_{2}$.

Proposition 1. Let $f \in S_{n}$, let $p$ be a prime number. The equation $x^{p}=f$ has an exact solution if and only if for any $k \in \mathbf{N}$ the number of $k p$-cycles in $f$ is divisible by $p$.

Proof. $\Rightarrow$ If $x^{p}=f$, the $m$-cycles in $x$ with $(p, m)=1$ become $m$-cycles in $f$. For the $k p$-cycles in $x$, we obtain $p$ cycles of length $k$ in $f$. Therefore, the $k p$-cycles in $f$ can be obtained only by the $k p^{2}$-cycles in $x$, so, in $f$ we will have $p$ cycles of length $k p$ for every $k p^{2}$-cycle in $x$.

$\Leftarrow$ Given the permutation $f$ we will construct a permutation $x$ that satisfies the equation. Suppose that the permutation $f$ has the following cyclic representation: $f=C_{1} \ldots C_{h} D_{1} \ldots D_{i}$, where $C_{i}$ are all $i$-cycles, with $i$ relatively prime to $p$ and $D_{k}$ are all cycles of length $k p$. For any $C_{r}$ in $f$ we can write $C_{r}^{\alpha_{r}}$ in $x$ where $\alpha_{r} p=1 \bmod r$. Now, as for any $k$, the number $m$ of $k p$-cycles is divisible by $p$, we can divide the cycles $D_{k}$ in disjoint groups of size $p, D_{k}=d_{1} d_{2} \ldots d_{m / p}$. For each group $d_{l}$

$$
d_{l}=\left(a_{0}^{0}, a_{1}^{0}, \cdots, a_{k p-1}^{0}\right)\left(a_{0}^{1}, a_{1}^{1}, \cdots, a_{k p-1}^{1}\right) \cdots\left(a_{0}^{p-1}, a_{1}^{p-1}, \cdots, a_{k p-1}^{p-1}\right)
$$

in $f$ we can take

$x_{l}=\left(a_{0}^{0}, a_{0}^{1}, \cdots, a_{0}^{p-1}, a_{1}^{0}, a_{1}^{1}, \cdots, a_{1}^{p-1}, a_{2}^{0}, \cdots, a_{2}^{p-1}, \cdots, a_{k p-1}^{0}, a_{k p-1}^{1}, \cdots, a_{k p-1}^{p-1}\right)$,

as the corresponding cycle of the permutation $x$.

Proposition 2. Let $p$ be a prime number, let $f \in S_{n}$, then there exist permutations $\tilde{f}, g \in S_{n}$, such that $g^{p}=\tilde{f}$, and $h(\tilde{f}, f) \leq \frac{2 \sqrt{2}(p-1)}{\sqrt{p n}}$.

Proof. In order to prove the proposition it is enough to construct $\tilde{f}$ satisfying Proposition 1. Let the permutation $f$ has the following cyclic representation: $f=$ $C_{1} \ldots C_{h} D_{1} \ldots D_{j}$, where the $C_{i}$ are all $i$-cycles, with $(p, i)=1$ and $D_{i}$ are all $i p$ cycles. Let $n_{o}$ be the number of all elements that belongs to the cycles $C_{1}, \cdots, C_{h}$, let $m_{i}$ be the number of all $i p$-cycles. Because some of the $m_{i}$ can be zero, we consider the following set $S:=\left\{i \mid m_{i} \neq 0\right\}$.

By Proposition 1 , in order to construct the permutation $\tilde{f}$, we only need to change some cycles in $D_{i}$. We have $m_{i}=\alpha_{i} p+r_{i}, 0 \leq r_{i}<p$ and construct $\tilde{f}$ equal to $f$ but delete one element for the last $r_{i} i p$-cycles, and make it fixed point $\left(\left(a_{1}, a_{2}, \ldots, a_{i p-1}, a_{i p}\right) \rightarrow\left(a_{1}, a_{2}, \ldots, a_{i p-1}\right)\left(a_{i p}\right)\right)$. Then the distance between $f$ and $\tilde{f}$ will be

$$
h(f, \tilde{f})=\frac{2 \sum_{i \in S} r_{i}}{n} \leq \frac{2(p-1)|S|}{n},
$$


So, we only need to estimate $k=|S|$ for $n$ fixed. To make the estimation let us put in order $S=\left\{s_{1}, s_{2}, \ldots, s_{k}\right\}$, where $1 \leq s_{1}<s_{2}<\ldots<s_{k}$. It follows that $s_{i} \geq i$. Now

$$
n=n_{0}+p \sum_{i \in S} m_{i} i \geq p \sum_{i=1}^{k} s_{i} \geq p \sum_{i=1}^{k} i=p \frac{k(k+1)}{2}>p \frac{k^{2}}{2} .
$$

So, $|S|=k<\sqrt{2 n / p}$ and the proposition follows.

Proof of Theorem 2 First Part. Let $V$ be a finite set of finite words in $x_{1}, x_{2}, \ldots, x_{k}$ that represent each element of the group $G$. Without loss of generality we will assume that $\left\{x_{1}^{ \pm 1}, x_{2}^{ \pm 1}, \ldots, x_{n}^{ \pm 1}\right\} \subseteq V$. For $v_{1}, v_{2} \in V$, the juxtaposed product $v_{1} \cdot v_{2}$ form a finite word, which does not necessarily belong to $V$. By the method of insertion and deletion of trivial and defining relators of $G$, the word $v_{1} \cdot v_{2}$ can be reduced to a word $v_{1,2} \in V$. Let $m$ be the maximum length of the words appearing during these reduction processes for all triples of words $v_{1}, v_{2}, v_{1,2} \in V$, $v_{1} v_{2}=v_{1,2}$ in $G$.

Let $f=\left\langle f_{1}, f_{2}, \ldots, f_{k}\right\rangle \in S_{n}^{k}$ be an $\epsilon$ solution of System 2. We think that the language of graphs is the most appropriate to expose our proof. So, we can consider $f_{1}, f_{2}, \ldots, f_{k} \in S_{n}$ as an edge-colored graph $\Gamma(f)$ with vertex set $V(\Gamma)=\{n\}$ and edge set $E(\Gamma)=E_{1} \cup E_{2} \cup \ldots \cup E_{k}$, where $E_{i}=\left\{\left(a,(a) f_{i}\right), a \in\{n\}\right\}$ is the edges of color $i$. Let $N(a)$ be the $m$-neighborhood of a vertex $a$ in $\Gamma$, where $m$ is the maximum length defined above. We call $a \in\{n\}$ to be a good vertex iff for any $c \in N(a) f$ satisfies System 2 in $c$ :

$$
(c) w_{i}\left(f_{1}, \ldots, f_{k}\right)=(c) u_{i}\left(f_{1}, \ldots, f_{k}\right), \quad i=1, . ., r .
$$

A vertex is bad if it is not good.

Claim 1. Let $a \in\{n\}$ be a good vertex, then $(c) x_{i}=(c) f_{i}$, for $c \in N(a)$ defines an action of $G=\left\langle x_{1}, \ldots, x_{k} \mid w_{i}\left(x_{1}, \ldots, x_{k}\right)=u_{i}\left(x_{1}, \ldots, x_{k}\right), i=1, \ldots, r\right\rangle$ on $N(a)$. It implies that any $c \in N(a)$ is also a good vertex, $N(c)=N(a)$ and the set of good vertexes is disconnected from the set of bad vertexes.

Proof. Indeed, let $p_{1}=v_{1} v_{2}, p_{2}, \ldots, p_{n}=v_{1,2}$ be the reduction from $v_{1} v_{2}$ to $v_{1,2}$. Then, $(a) p_{1}(f)=(a) p_{2}(f)=\ldots=(a) p_{n}(f)$ by the definition of good vertexes and the claim follows.

We may construct $(a) \tilde{f}_{i}=(a) f_{i}$ if $a$ is good vertex and $(a) \tilde{f}_{i}=a$ if $a$ is bad vertex. It follows that $\tilde{f}_{i}$ satisfy System 2 , because the set of bad vertexes is separated from the set of good vertexes. So it is enough to show that the set of bad vertexes is small. Let $M=\left\{a \in\{n\} \mid a u_{i}(f) \neq a w_{i}(f)\right.$ for some $\left.i\right\}$, it is clear 
that $|M| \leq \epsilon k n$. Then the set of bad points is $M^{*}=\bigcup_{b \in M} N(b)$, so

$$
\left|M^{*}\right| \leq \sum_{b \in M}|N(b)| \leq \epsilon k n\left(1+k \frac{\left((2 k-1)^{m-1}-1\right)}{k-1}\right)
$$

So $h\left(f_{i}, \tilde{f}_{i}\right) \leq C \epsilon$, where $C$ depends only on the group $G$.

Proof of the Second Part of Theorem 2. For a group $X$ we will denote by $e_{X}$ the unity in $X$, some times we will write just $e$. For the second part of the theorem 2 we recall the following definitions.

Definition 2. Let $G$ be a group, $F \subseteq G$ be a finite subset, $\epsilon \geq 0$, and $\alpha>0$. An $(F, \epsilon, \alpha)$-representation in $\left(S_{n}, h\right)$ is a map $\phi: F \rightarrow S_{n}$ with the following properties

(1.) For any two elements $a, b \in F$, with $a \cdot b \in F, h(\phi(a) \phi(b), \phi(a \cdot b))<\epsilon$

(2.) If $e \in F$, then $\phi(e)=i d$

(3.) For any $a \neq e, h(\phi(a), i d)>\alpha$

Definition 3. The group $G$ is sofic if there exists $\alpha_{0}>0$ such that for any finite set $F \subseteq G$ and for any $\epsilon>0$ there exists an $\left(F, \epsilon, \alpha_{0}\right)$-representation in $\left(S_{n}, h\right)$.

Definition 4. A group $G$ is residually finite iff for any $g \in G, g \neq e_{G}$, there exists a homomorphism $\phi$ to a finite group $H$ such that $\phi(g) \neq e_{H}$.

We need the following lemma:

Lemma 1. If $d(\cdot, \cdot)$ is a bi-invariant metric, and $d\left(x_{i}, y_{i}\right) \leq \delta_{i}, i=1, \ldots, r$, then $d\left(x_{1} \cdots x_{r}, y_{1} \cdots y_{r}\right) \leq \sum_{i=1}^{r} \delta_{i}$

Proof. By induction and by the bi-invariance of the metric, we have that

$$
\begin{aligned}
d\left(x_{1} \cdots x_{r+1}, y_{1} \cdots y_{r+1}\right) \leq & d\left(x_{1} \cdots x_{r+1}, y_{1} \cdots y_{r} \cdot x_{r+1}\right) \\
& +d\left(y_{1} \cdots y_{r} \cdot x_{r+1}, y_{1} \cdots y_{r} \cdot y_{r+1}\right) \\
\leq & d\left(x_{1} \cdots x_{r}, y_{1} \cdots y_{r}\right)+d\left(x_{r+1}, y_{r+1}\right) \\
\leq & \sum_{i=1}^{r} \delta_{i}+\delta_{r+1}=\sum_{i=1}^{r+1} \delta_{i}
\end{aligned}
$$

In order to proof the second part of theorem 2, we will prove the following.

Proposition 3. If $G=\left\langle x_{1}, \ldots, x_{k}\right| w_{i}\left(x_{1}, \ldots, x_{k}\right)=u_{i}\left(x_{1}, \ldots, x_{k}\right), i=$ $1, \ldots, r\rangle$ is sofic and System 2 is stable, then $G$ is residually finite. 
Proof. Let $p(\bar{x})$ be any word in $G, p \neq e_{G}$. We need to construct a homomorphism $\phi$ to a finite group, such that $\phi(p) \neq e$. We denote by $V^{*}$ the set of the words $w_{i}\left(x_{1}, \ldots, x_{k}\right), u_{i}\left(x_{1}, \ldots, x_{k}\right), i=1, \ldots, r$ and all its subwords and let $p^{*}$ denote the set of all subwords of $p$. Let $F$ be the following set: $F:=\left\{1, x_{1}, x_{1}^{-1}, \ldots, x_{k}, x_{k}^{-1}\right\} \cup V^{*} \cup p^{*}$. It is clear, that $F$ is finite and for any word in $F$ all its subwords belongs to $F$. As the group $G$ is sofic, there exists $\alpha>0$ such that for any $\epsilon>0$ there exists an $(F, \epsilon, \alpha)$-representation $\varphi$. We denote $a_{i}:=\varphi\left(x_{i}\right)$.

Claim 2. For any word $v(\bar{x}) \in F, h(\varphi(v(\bar{x})), v(\bar{a}))<(2|v(\bar{x})|-1) \epsilon$

Proof. As $h\left(\varphi\left(x_{i}^{-1}\right), a_{i}^{-1}\right)<\epsilon$, by induction and Lemma 1 we have

$$
\begin{aligned}
h\left(\varphi\left(x_{i}^{ \pm 1} v(\bar{x})\right), a_{i}^{ \pm 1} v(\bar{a})\right) & <h\left(\varphi\left(x_{i}^{ \pm 1} v(\bar{x})\right), \varphi\left(x_{i}^{ \pm 1}\right) \varphi(v(\bar{x}))\right) \\
& +h\left(\varphi\left(x_{i}^{ \pm 1}\right) \varphi(v(\bar{a})), a_{i}^{ \pm 1} v(\bar{a})\right) \\
& \leq \epsilon+(\epsilon+(2|v(\bar{x})|-1) \epsilon)=(2(1+|v(\bar{x})|)-1) \epsilon .
\end{aligned}
$$

So, $a_{i}=\varphi\left(x_{i}\right)$ is an $\epsilon^{*}$-solution of the system 2 with $\epsilon^{*}=\max \left\{2\left(\left|w_{i}\right|+\mid\right.\right.$ $\left.\left.u_{i} \mid\right) \epsilon\right\}$. As the system 2 is stable, we can find an exact solution $b_{1}, \ldots, b_{n}$ of the system 2 , with $h\left(a_{i}, b_{i}\right) \leq \delta_{\epsilon^{*}}$ for any $i$. Then $\phi\left(x_{i}\right)=b_{i}$, can be extended to a homomorphism $G \rightarrow S_{n}$.

Claim 3. For any word $w \in F, w \neq e_{G}$ one has $h(\phi(w(\bar{x})), i d) \geq \alpha-\delta_{\epsilon^{*}}^{*} \mid$ $w(\bar{x})|-2| w(\bar{x}) \mid \epsilon$

Proof. Because $\phi\left(x_{i}\right)$ is a homomorphism $\phi(w(\bar{x}))=w(\bar{b})$, and by Lemma 1, $h(w(\bar{a}), w(\bar{b})) \leq \delta_{\epsilon^{*}}^{*}|w(\bar{x})|$ then

$$
\begin{aligned}
h(\phi(w(\bar{x})), \varphi(w(\bar{x}))) & \leq h(\phi(w(\bar{x})), w(\bar{b}))+h(w(\bar{b}), \varphi(w(\bar{x}))) \\
& \leq h(w(\bar{b}), w(\bar{a}))+h(w(\bar{a}), \varphi(w(\bar{x}))) \\
& \leq \delta_{\epsilon^{*}}^{*}|w(\bar{x})|+2|w(\bar{x})| \epsilon
\end{aligned}
$$

as $h(\varphi(w(\bar{x})), i d) \leq h(\phi(w(\bar{x})), \varphi(w(\bar{x})))+h(\phi(w(\bar{x})), i d)$, then

$$
h(\phi(w(\bar{x})), i d) \geq \alpha-\delta_{\epsilon^{*}}^{*}|w(\bar{x})|-2|w(\bar{x})| \epsilon=\alpha_{0}
$$

So, $\phi(p) \neq i d$ for sufficiently small $\epsilon>0$. 


\section{ACKNOWLEDGMENT}

The authors would like to thank E. Gordon for useful discussions. The work was partially supported by CONACyT grant SEP-25750, and PROMEP grant UASLPCA-21.

\section{REFERENCES}

1. Choi, M. D. Almost commuting matrices need not be nearly commuting. Proc. Amer. Math. Soc. 102, 3 (1988), 529-533.

2. Deza, M., and Huang, T. Metrics on permutations, a survey. http://citeseer.ist.psu.edu/ cache/90004.html.

3. Elek, G., and Szabó, E. Hyperlinearity, essentially free action and $1^{2}$-invariants. the sofic property. Math. Ann. 332, 2 (2005), 421-441.

4. Farah, I. All automorphisms of the calkin algebra are inner., 2007. preprint 0705.3085, http://arxiv.org/.

5. Gorenstein, D., Sandler, R., and Mills, W. H. On almost-commuting permutations. Pacific J. Math., 12 (1962), 913-923.

6. Gromov, M. Endomorphisms of symbolic algebraic varieties. J. Eur. Math. Soc., 1 (1999), 109-197.

7. Kropholer, P. Baumslag-soliter groups and some other groups of cohomological dimension two. Comment. Math. Helvetici, 65 (1990), 547-558.

8. Lin, H. Almost commuting selfadjoint matrices and applications. In Operator algebras and their applications (Waterloo, ON, 1994/1995), vol. 13 of Fields Inst. Commun. Amer. Math. Soc., Providence, RI, 1997, pp. 193-233.

9. Radulescu, F. The von neumann algebra of the non-residually finite baumslag group $\left\langle a, b \mid a b^{3} a^{-1}=b^{2}\right\rangle$ embeds into $\mathbf{R}^{\omega}$, 2002. preprint 0004172, http://xxx.lanl.gov/.

10. Tristan, I. A. Grupo de baumslag-solitar bs(2,3) es un grupo sofico, 2007.

11. Voiculescu, D. Asymptotically commuting finite rank unitary operators without commuting approximants. Acta Sci. Math. (Szeged), 45(1-4) (1983), 429-431.

12. Weiss, B. Sofic groups and dynamical systems. Ergodic theory and harmonic analysis, 62 (2000), 350-359.

Lev Glebsky and Luis Manuel Rivera

\section{IICO-UASLP.}

Av. Karakorum 1470, Lomas 4ta Session,

San Luis Potosi, SLP 7820,

Mexico

E-mail: glebsky@ cactus.iico.uaslp.mx 